\title{
Optimal Proliferation Rate in a Cell Division Model
}

\author{
P. Michel $^{1}$ \\ Department of Mathematics and Informatics, Institut Camille Jordan, \\ Ecole centrale de Lyon, 36 avenue Guy de Collongue, 69134 Ecully, FRANCE
}

\begin{abstract}
We consider a size structured cell population model where a mother cell gives birth to two daughter cells. We know that the asymptotic behavior of the density of cells is given by the solution to an eigenproblem. The eigenvector gives the asymptotic shape and the eigenvalue gives the exponential growth rate and so the Maltusian parameter. The Maltusian parameter depends on the division rule for the mother cell, i.e., symmetric (the two daughter cells have the same size) or asymmetric. We use a min-max principle and a differentiation principle to find the variation of the first eigenvalue with respect to a parameter of asymmetry of the cell division. We prove that the symmetrical division is not always the best fitted division, i.e., the Maltusian parameter may be not optimal.
\end{abstract}

Key words: cell division, long time asymptotic, eigenvalue, min-max, variation, asymmetry. AMS subject classification: 35P05, 92B05, 93B60

\section{Introduction}

General models of cell division are known for a long time. Although the most classical case is division into two equal new cells, it is now well established that this is not always the case (see $[29,18,25,1,24,28])$. In particular in hematopoiesis (see [7, 15] or [30] for thymic lymphoblasts), in the large class of budding yeasts (see[17, 6, 2, 27]), E.Coli or for some other bacteria [9] like Physcomitrella protoplast, division is not always symmetric and a mother cell can give birth to a bigger and a smaller cells. The goal of the present paper is to propose a possible explaination for the existence of different way of cell division (symmetric or asymmetric) based on adaptive dynamic. In some cases, the symmetric division is not the best fitted. The natural model to study

\footnotetext{
${ }^{1}$ Corresponding author. Email: philippe.michel@ec-lyon.fr
} 
it is a cell division model (see $[10,20]$ ) in which the density of cells $n(t, y)$ is structured by their size $y$ and the evolution is described by the master equation

$$
\frac{\partial}{\partial t} n(t, y)+\frac{\partial}{\partial y} n(t, y)+B(y) n(t, y)=\frac{1}{\sigma} B\left(\frac{y}{\sigma}\right) n\left(t, \frac{y}{\sigma}\right)+\frac{1}{1-\sigma} B\left(\frac{y}{1-\sigma}\right) n\left(t, \frac{y}{1-\sigma}\right),
$$

where a cell of size $y$ gives birth to a cell of size $y \sigma$ and another one of size $(1-\sigma) y$, with $\sigma \in] 0,1$. A similar model also arises to describe fragmentation in physics [13,19] and the growth term $\partial_{y} n$ arises after rescalling $[5,11]$. The division part of this equation can be understood as follows. The cells of size $y$ are produced from the cells of size $y / \sigma$ (resp. $y /(1-\sigma)$ ). The number of cells which size belongs to $[y, y+\delta y]$ that appear after the division are $\frac{\delta y}{\sigma} B\left(\frac{y}{\sigma}\right) n\left(t, \frac{y}{\sigma}\right)$ (resp. $\frac{\delta y}{1-\sigma} B\left(\frac{y}{1-\sigma}\right) n\left(t, \frac{y}{1-\sigma}\right)$ ). We have then the population balance equation (1.1). We know from the general theory (see $[22,21]$ ) that the asymptotic behavior of such an evolution equation is given by the rate $\lambda$, the eigenvalue of the following eigenproblem

$$
\left\{\begin{array}{l}
\frac{\partial}{\partial y} N(y)+[\lambda+B(y)] N(y)=\frac{1}{\sigma} B\left(\frac{y}{\sigma}\right) N\left(\frac{y}{\sigma}\right)+\frac{1}{1-\sigma} B\left(\frac{y}{1-\sigma}\right) N\left(\frac{y}{1-\sigma}\right), \\
-\frac{\partial}{\partial y} \phi(y)+\lambda \phi(y)=B(y)[\phi(\sigma y)+\phi((1-\sigma) y)-\phi(y)], \\
N(y=0)=0, \quad N, \phi \geq 0, \quad \int_{0}^{\infty} N(y) \phi(y) d y=1, \quad \phi(0)=1 .
\end{array}\right.
$$

More precisely, we know from general relative entropy method [21, 26], that there exists a constant $C$ only depending on the initial condition $n(0,$.$) such that :$

- $C N(.) e^{\lambda t}$ is a solution to (1.1),

- $\phi e^{-\lambda t}$ is a solution to the backward equation of (1.1), or similarly, $\phi$ is a solution to the dual eigenproblem,

- finally, we have the convergence in the weighted space $L^{1}([0, \infty[, \phi d y)$ with the norm $\|f\|:=$ $\int|f(y)| \phi(y) d y$

$$
\lim _{t \rightarrow \infty} n(t, .) e^{-\lambda t}=C N(.) \text { in } L^{1}([0, \infty[, \phi d y) .
$$

Consequently, the dynamics of the density $n(t, y)$ is strongly related to the eigenvalue in (1.2) that gives an invasive parameter of the population (and thus the fitness of the population). We use a min-max method $[3,4,14]$ and a differentiation method to study the variation of the first eigenvalue $\lambda$ with respect to the asymmetry parameter $\sigma$. We prove that when a cell divides early then the asymmetric division gives a better growth, i.e., a larger first eigenvalue. On the contrary, for late division, asymmetric division is better.

This paper is organized as follows. Firstly, in Section 2, we give the main results on the variation of the invasive parameter (first eigenvalue in (1.2)) with respect to the asymmetry of the division. We show that the convexity of the dual eigenfunction $\phi$ is related to this problem of variation. Then, in Section 3, we show that a min-max method and a differentiation method can be used to 
study the variation of the invasive parameter (first eigenvalue) with respect to the parameter of cell division asymmetry $\sigma$ in (1.1). In Section 4, we consider birth rate $B$ such that we obtain directly the convexity of the dual eigenfunction. In Section 5, we extend the results found in Section 2 to more general cell division models. Finally, in the last section, we give some simple applications of the main methods.

\section{Main results}

The purpose of this section is to show that the symmetric division is not necessarily the best fitted division. We study two different cases, when the birth rate $B$ has a compact support separated from $y=0$,

$$
\text { Supp } B \subset[a, b], \quad a>b / 2,
$$

or Supp $B$ contains $y=0$. In this case we suppose that

$$
\left.\operatorname{Supp} B=[0, b], \quad \frac{\lambda(\sigma)(\lambda(\sigma)-B(0))}{B(0)}<\frac{B^{\prime}(y)}{B(y)} \leq 0, \quad \forall y \in\right] 0, b \sigma_{0}\left[, \quad \forall \sigma \in\left[1 / 2, \sigma_{0}[,\right.\right.
$$

with $\sigma_{0} \in[1 / 2,1[, \lambda(\sigma)$ the first eigenvalue $\lambda$ in (1.2) where $\sigma$ is the asymmetric parameter. We notice that $B(y)=1_{[0, b]}$ satisfies assumption (2.2). This condition means that the cells begin to divide early (at size $y=0$ ) and the birth rate decreases.

Theorem 2.1. Assume that Supp B is compact and (2.1) holds. Then

$$
\frac{d}{d \sigma} \lambda(\sigma) \leq 0, \quad \forall \sigma \in[1 / 2, a / b[
$$

Moreover, we have

$$
\sup _{\sigma \in] 1-a / b, a / b[} \lambda(\sigma)=\lambda(1-a / b) .
$$

Theorem 2.1.Assume that $b>0, \sigma_{0} \in[1 / 2,1[$ and (2.2) holds. Then

$$
\frac{d}{d \sigma} \lambda(\sigma) \geq 0, \quad \forall \sigma \in\left[1 / 2, \sigma_{0}[\right.
$$

Moreover, we have

$$
\sup _{\sigma \in] 1-\sigma_{0}, \sigma_{0}[} \lambda(\sigma)=\lambda(1 / 2)
$$

\section{Proof of Theorems 2.1-2.2}

First, we will recall some results on the existence of a solution to the eigenproblem. Then, we differentiate the first eigenvalue $\lambda(\sigma)$ with respect to the parameter $\sigma$ to study the variation of $\lambda(\sigma)$. We notice that the variation of the first eigenvalue is directly linked to the properties of the 
dual eigenfunction $\phi_{\sigma}$ to be concave or convex. Finally, we prove that under the assumptions of Theorems 2.1-2.2, we can directly prove the convexity or concavity of the dual eigenfunction.

Step 1. Existence and uniqueness of the solution to eigenproblem (1.2)

The eigenproblem (1.2) associated to the cell division model can be written with more compact notations

$$
\mathcal{L}_{\sigma}^{*}\left(\phi_{\sigma}\right)=\lambda(\sigma) \phi_{\sigma}, \quad \mathcal{L}_{\sigma}\left(N_{\sigma}\right)=\lambda(\sigma) N_{\sigma}, \quad \int_{0}^{\infty} N_{\sigma}(y) \phi_{\sigma}(y) d y=1
$$

where $L_{w}^{1}\left(\left[0, \infty[):=L^{1}([0, \infty[,(1+B(y))(1+y) d y)\right.\right.$,

$\mathcal{L}_{\sigma}(f):=-\frac{\partial f}{\partial y}()-.B() f.(t,)+.\frac{1}{\sigma} B\left(\frac{\dot{\sigma}}{\sigma}\right) f\left(\frac{\dot{\sigma}}{\sigma}\right)+\frac{1}{1-\sigma} B\left(\frac{\cdot}{1-\sigma}\right) f\left(\frac{\cdot}{1-\sigma}\right), f \in L_{w}^{1}([0, \infty[)$,

and the dual operator,

$$
\mathcal{L}_{\sigma}^{*}(g):=\frac{\partial g}{\partial y}(.)-B(.) g(t, .)+B(.)(g(. \sigma)+g(.(1-\sigma))), g \in L_{w}^{1}\left(\left[0, \infty[)^{*} .\right.\right.
$$

Remark We notice that it is enough to consider $\sigma \in[1 / 2,1[$, since, using the symmetry of (1.2), we have $\lambda(\sigma)=\lambda(1-\sigma)$.

Here, we cannot expect to find an exact solution as in the McKendrick-VonFoerster model for instance. Nevertheless, we have the following lemma.

Lemma 2.3.Under the assumption (2.1) (resp. (2.2)), there exists a solution $\left(N_{\sigma}, \lambda(\sigma), \phi_{\sigma}\right)$ to (2.5)-(2.7). Moreover, we have

$$
\forall \sigma \in] 0,1\left[, \quad \exists C_{\sigma}<\infty:\left\{\begin{array}{l}
N_{\sigma}(y) \leq C_{\sigma} e^{-\lambda(\sigma) y}, \\
\phi_{\sigma}(y) \leq C_{\sigma}(1+y), \\
\left|\frac{\partial}{\partial y} \phi_{\sigma}(y)\right| \leq C_{\sigma}(B(y)+1)(1+y) .
\end{array}\right.\right.
$$

We refer to [22] for the proof of this result.

\section{Step 2. The differentiation method.}

Lemma 2.4.The function $\sigma \mapsto \lambda(\sigma)$ is well defined on $] 0,1[$ and is differentiable. Moreover, we have

$$
\frac{\partial}{\partial \sigma} \lambda(\sigma)=\int_{0}^{\infty} B(y)\left[\frac{\partial}{\partial y} \phi_{\sigma}(\sigma y)-\frac{\partial}{\partial y} \phi_{\sigma}((1-\sigma) y)\right] N_{\sigma} d y
$$

We prove this lemma in Section 3. (as an application of Lemma 3.2) and using the same method, we find :

Lemma 2.5.The following equality holds :

$$
\left(\frac{\partial^{2}}{\partial \sigma^{2}} \lambda(\sigma)\right)_{\sigma=1 / 2}=2 \int_{0}^{\infty} B(y) y^{2} \frac{\partial^{2}}{\partial y^{2}} \phi_{1 / 2}(y / 2) N_{1 / 2}(y) d y
$$


Proof. We use Lemma 2.4 and the same method to prove that $\frac{\partial^{2}}{\partial \sigma^{2}} \lambda(\sigma)$ exists and satisfies the above equality.

Now, we can give a direct corollary of Lemma 2.4. We suppose that

$$
\begin{gathered}
\sigma_{0} \in[1 / 2,1[, \\
K=\operatorname{Supp} B \subset[0, \infty[.
\end{gathered}
$$

Corollary 2.6. Assume that $\phi_{\sigma_{0}}$ is a convex (resp. concave) function on $\left[1-\sigma_{0}, \sigma_{0}\right]$.Conv Hull $(K)$. Then we find that

$$
\left(\frac{\partial}{\partial \sigma} \lambda_{\sigma}\right)_{\sigma=\sigma_{0}} \geq 0 \quad\left(\operatorname{resp} \cdot\left(\frac{\partial}{\partial \sigma} \lambda_{\sigma}\right)_{\sigma=\sigma_{0}} \leq 0\right) .
$$

Proof. Indeed, using Lemma 2.4 and that $\phi_{\sigma_{0}}$ is a convex function, we find that $\frac{\partial}{\partial y} \phi_{\sigma_{0}}$ is increasing. Since $\sigma_{0} \geq\left(1-\sigma_{0}\right)$, we have $\left(\frac{\partial}{\partial \sigma} \lambda_{\sigma}\right)_{\sigma=\sigma_{0}} \geq 0$.

Remark The strict convexity (resp. strict concavity) of $\phi_{\sigma_{0}}$ on $K$ implies $\frac{\partial}{\partial \sigma} \lambda>0$ (resp. $\left.\frac{\partial}{\partial \sigma} \lambda<0\right)$.

Thus, we reduce the problem of the variation of $\lambda(\sigma)$ with respect to $\sigma$ to a problem on $\phi_{\sigma}$. In the next section we prove that under some assumptions on $B$, the dual eigenfunction $\phi_{\sigma}$ is a convex or concave function.

Step 3. Convexity of the dual eigenfunction $\phi_{\sigma}$

When the birth rate has a compact support, the following result holds.

Theorem 2.7. Assume that (2.1) (resp. (2.2) with $\sigma_{0} \in[1 / 2,1[$ ) holds. Then for all $\sigma \in] 1-$ $a / b, a / b\left[\left(\right.\right.$ resp. $\left.\sigma \in\left[1 / 2, \sigma_{0}\right]\right), \phi_{\sigma}$ is a strictly convex (resp. strictly concave) function.

We prove Theorem 2.7 in section 4.

\section{Step 4. Conclusion}

We can now prove the main Theorems 2.1-2.2 on the variation of the eigenvalue $\lambda(\sigma)$ with respect to the asymmetry parameter $\sigma$. Indeed, using Corollary 2.6 and the results proved in Section 2., we get directly Theorems 2.1-2.2.

\section{Main methods}

Formally, we have a family of linear bounded operators (and their dual operators) which depend on a parameter $p \in I$, where $I \subset]-\infty, \infty[$ is an interval,

$$
\mathcal{L}_{p}: \mathcal{B} \mapsto \mathcal{C}, \quad \mathcal{L}_{p}^{*}: \mathcal{C}^{*} \mapsto \mathcal{B}^{*}
$$

$B$ and $C$ are Banach spaces of real functions (in duality $\langle.,$.$\rangle with B^{*}$ and $C^{*}$ ) such that

$$
\left.\forall p \in I \quad \exists\left(N_{p}, \lambda(p), \phi_{p}\right) \in \mathcal{B}_{+} \times\right]-\infty, \infty\left[\times \mathcal{C}_{+}^{*} \quad: \quad \mathcal{L}_{p}\left(N_{p}\right)=\lambda(p) N_{p}, \mathcal{L}_{p}^{*}\left(\phi_{p}\right)=\lambda(p) \phi_{p}\right.
$$


where $\mathcal{B}_{+}\left(\operatorname{resp} \mathcal{C}_{+}^{*}\right)$ is the positive cone of $\mathcal{B}\left(\operatorname{resp} \mathcal{C}^{*}\right)$ and

$$
\forall p \in I \quad\left\langle\phi_{p}, N_{p}\right\rangle=1 .
$$

We will study the variation of $\lambda(p)$ with respect to $p$. We notice that the variation of $N_{p}, \phi_{p}$ in Banach spaces of real functions makes the problem rather complex.

Our purpose is to find the variation of the first eigenvalue in a general eigenvalue problem (3.1)-(3.3). To study the variation of the first eigenvalue, we develop two points of view, one which favors the min-max principle and the other which uses the differentiation of the eigenvalue (and eigenproblem). Even if the second one needs the operators in the eigenproblem to be more regular, it gives better results in some problems as the cell division one.

Min-max method: We have the following principle

Lemma 3.1. Assume that there exists a solution $\left(N_{p}, \lambda(p), \phi_{p}\right)$ to (3.1)-(3.3). Then we have for all $p \in I$,

$$
\lambda(p)=\max _{f \in \Lambda} \min _{g \in \Lambda^{*}} \frac{\left\langle g, \mathcal{L}_{p} f\right\rangle}{\langle g, f\rangle},
$$

where $\Lambda \subset \mathcal{B}_{+}$which satisfies $N_{p} \in \Lambda, \phi_{p} \in \Lambda^{*} \subset \mathcal{C}_{+}^{*}$ and $\langle g, f\rangle>0$ for all $f \in \Lambda$ and $g \in \Lambda^{*}$. Moreover, if $\mathcal{L}_{p}$ is monotone with respect to $p \in I$ then so is $\lambda(p)$. More precisely, we have

$$
\forall p \leq q \in J, \quad \forall(f, g) \in \Lambda \times \Lambda^{*} \quad\left\langle g, \mathcal{L}_{p}(f)\right\rangle \leq\left\langle g, \mathcal{L}_{q}(f)\right\rangle \Longrightarrow \quad \lambda(p) \leq \lambda(q) .
$$

Finally, if $\mathcal{L}_{p}$ is continuous with respect to $p$,

$$
\forall(f, g) \in \Lambda \times \Lambda^{*} \quad \lim _{p \rightarrow q}\left|\left\langle g, \mathcal{L}_{p}(f)-\mathcal{L}_{q}(f)\right\rangle\right|=0,
$$

then $\lambda(p)$ is continuous with respect to $p$.

Differentiation method : We have the following principle

Lemma 3.2. Assume, there exists $\left(N_{p}, \lambda(p), \phi_{p}\right)$ solution to (3.1)-(3.3) and

$$
\forall q \in \stackrel{\circ}{J}, \quad \lim _{p \rightarrow q \in \dot{J}} N_{p}=N_{q}, \text { strongly in } \mathcal{B},
$$

$$
\left.\forall q \in \stackrel{\circ}{J} \exists \partial_{p} \mathcal{L}_{p}^{*}\right|_{p=q} \in \mathcal{L}\left(\mathcal{C}^{*}, \mathcal{B}^{*}\right) \quad: \quad \forall f \in \Lambda, \quad \lim _{p \rightarrow q \in J}\left\langle\frac{\mathcal{L}_{p}^{*}-\mathcal{L}_{q}^{*}}{p-q} \phi_{q}, f\right\rangle=\left\langle\left.\partial_{p} \mathcal{L}_{q}^{*}\right|_{p=q} \phi_{q}, f\right\rangle .
$$

Then $p \mapsto \lambda(p)$ is differentiable and we have for all $q \in \stackrel{\circ}{ } \subset I$,

$$
\left(\frac{d}{d p} \lambda\right)(q)=\left\langle\left.\partial_{p} \mathcal{L}_{q}^{*}\right|_{p=q} \phi_{q}, N_{q}\right\rangle
$$

Moreover, under the assumptions of Lemma 2.4, we have (2.9). 


\section{Proof of Lemma 3.1}

Since $N_{p} \in \Lambda$ and $\mathcal{L}_{p} N_{p}=\lambda(p) N_{p}$ then we have

$$
\forall g \in \Lambda^{*}, \quad \lambda(p)=\frac{\left\langle g, \mathcal{L}_{p} N_{p}\right\rangle}{\left\langle g, N_{p}\right\rangle} .
$$

Hence we obtain the inequality

$$
\lambda(p) \leq \max _{f \in \Lambda} \min _{g \in \Lambda^{*}} \frac{\left\langle g, \mathcal{L}_{p} f\right\rangle}{\langle g, f\rangle} .
$$

Now, assume there exists $f_{0} \in \Lambda$ such that

$$
\lambda(p)<\min _{g \in \Lambda^{*}} \frac{\left\langle g, \mathcal{L}_{p} f_{0}\right\rangle}{\left\langle g, f_{0}\right\rangle} .
$$

In particular, this inequality holds for $g=\phi_{p}$ and so we have

$$
\lambda(p)<\frac{\left\langle\phi_{p}, \mathcal{L}_{p} f_{0}\right\rangle}{\left\langle\phi_{p}, f_{0}\right\rangle}=\frac{\left\langle\mathcal{L}_{p}^{*} \phi_{p}, f_{0}\right\rangle}{\left\langle\phi_{p}, f_{0}\right\rangle}=\lambda(p)
$$

which is impossible. Thus (3.4) is satisfied. Now, assume that $\mathcal{L}_{p}$ is increasing in the sense of (3.5). Then we have the inequalities

$$
\left\langle g, \mathcal{L}_{p}(f)\right\rangle \leq\left\langle g, \mathcal{L}_{q}(f)\right\rangle
$$

and (using (3.4)),

$$
\min _{g \in \Lambda^{*}} \frac{\left\langle g, \mathcal{L}_{p} f\right\rangle}{\langle g, f\rangle} \leq \min _{g \in \Lambda^{*}} \frac{\left\langle g, \mathcal{L}_{q} f\right\rangle}{\langle g, f\rangle} \leq \lambda(q) .
$$

In particular, for $f=N_{p}$, we find :

$$
\lambda(p)=\min _{g \in \Lambda^{*}} \frac{\left\langle g, \mathcal{L}_{p} N_{p}\right\rangle}{\left\langle g, N_{p}\right\rangle} \leq \min _{g \in \Lambda^{*}} \frac{\left\langle g, \mathcal{L}_{q} N_{p}\right\rangle}{\left\langle g, N_{p}\right\rangle} \leq \lambda(q) .
$$

Finally, if $\mathcal{L}_{p}$ is continuous with respect to $p$, then using the min-max property, we have,

$$
\frac{\left\langle g, \mathcal{L}_{p} f\right\rangle}{\langle g, f\rangle} \leq \frac{\left\langle g, \mathcal{L}_{q} f\right\rangle}{\langle g, f\rangle}+\epsilon(p, q)
$$

where $\epsilon(p, q) \rightarrow_{p \rightarrow q} 0$. Thus, we obtain that

$$
\min _{g \in \Lambda^{*}} \frac{\left\langle g, \mathcal{L}_{p} f\right\rangle}{\langle g, f\rangle} \leq \min _{g \in \Lambda^{*}} \frac{\left\langle g, \mathcal{L}_{q} f\right\rangle}{\langle g, f\rangle}+\epsilon(p, q) \leq \lambda(q)+\epsilon(p, q),
$$

and so

$$
\lambda(p) \leq \lambda(q)+\epsilon(p, q) .
$$


Similarly,

$$
\lambda(q) \leq \lambda(p)+\epsilon_{1}(p, q),
$$

where $\epsilon_{1}(p, q) \rightarrow_{p \rightarrow q} 0$. Therefore, we prove that $\lambda(p)$ is continuous with respect to $p$.

\section{Proof of Lemma 3.2}

For all $q, p \in \stackrel{\circ}{J}$, we have :

$$
\lambda_{q}=\left\langle\mathcal{L}_{q}^{*} \phi_{q}, N_{q}\right\rangle
$$

Thus, we find

$$
\lambda_{q}-\lambda_{p}=\left\langle\mathcal{L}_{q}^{*} \phi_{q}, N_{q}\right\rangle-\left\langle\mathcal{L}_{p}^{*} \phi_{p}, N_{p}\right\rangle
$$

We obtain, using the normalization (3.3), i.e. $\left\langle\phi_{q}, N_{q}\right\rangle=\left\langle\phi_{p}, N_{p}\right\rangle=1$, and $\left(\phi_{s}, N_{s}\right) \in \mathcal{C}^{*} \times$ $(\mathcal{B} \cap \mathcal{C})$

$$
\lambda_{q}-\lambda_{p}=\left\langle\mathcal{L}_{q}^{*} \phi_{q}-\mathcal{L}_{p}^{*} \phi_{q}, N_{p}\right\rangle+\left\langle\mathcal{L}_{q}^{*} \phi_{q}, N_{q}-N_{p}\right\rangle-\left\langle\mathcal{L}_{p}^{*} \phi_{p}-\mathcal{L}_{p}^{*} \phi_{q}, N_{p}\right\rangle,
$$

that gives, using (1.1), (3.2), and the duality between $\mathcal{L}_{q}$ and $\mathcal{L}_{q}^{*}$ :

$$
\lambda_{q}-\lambda_{p}=\left\langle\mathcal{L}_{q}^{*} \phi_{q}-\mathcal{L}_{p}^{*} \phi_{q}, N_{p}\right\rangle+\lambda_{q}\left\langle\phi_{q}, N_{q}-N_{p}\right\rangle-\lambda_{p}\left\langle\phi_{p}-\phi_{q}, N_{p}\right\rangle .
$$

Therefore, using the normalization (3.3), we find

$$
\lambda_{q}-\lambda_{p}=\left\langle\mathcal{L}_{q}^{*} \phi_{q}-\mathcal{L}_{p}^{*} \phi_{q}, N_{p}\right\rangle+\lambda_{q}\left\langle\phi_{q}, N_{q}-N_{p}\right\rangle-\lambda_{p}\left\langle\phi_{q}, N_{q}-N_{p}\right\rangle,
$$

So, we obtain

$$
\left(\lambda_{q}-\lambda_{p}\right)\left[1-\left\langle\phi_{q}, N_{p}-N_{q}\right\rangle\right]=\left\langle\mathcal{L}_{q}^{*} \phi_{q}-\mathcal{L}_{p}^{*} \phi_{q}, N_{p}\right\rangle .
$$

We notice that, for the moment, we only use (3.1)-(3.3). Hence (3.11) is always satisfied. Now, if we assume (3.7) and (3.8), then we have

$$
\begin{gathered}
\lim _{p \rightarrow q}\left[1-\left\langle\phi_{q}, N_{p}-N_{q}\right\rangle\right]=1, \\
\lim _{p \rightarrow q} \frac{\left\langle\mathcal{L}_{q}^{*} \phi_{q}-\mathcal{L}_{p}^{*} \phi_{q}, N_{p}\right\rangle}{q-p}=\left\langle\left.\partial_{p} \mathcal{L}_{q}^{*}\right|_{p=q} \phi_{q}, N_{p}\right\rangle,
\end{gathered}
$$

and so $\lambda(p)$ is differentiable and by passing to the limit, we find (3.9).

We prove Lemma 2.4. We have (see the equation (3.11)),

$$
\begin{aligned}
(\lambda(\sigma) & -\lambda(\sigma-\epsilon))\left[1-\int_{0}^{\infty}\left(\phi_{\sigma-\epsilon}(y)-\phi_{\sigma}(y)\right) N_{\sigma-\epsilon}(y) d y\right] \\
& =\int_{0}^{\infty}\left(\mathcal{L}_{\sigma}^{*}\left(\phi_{\sigma}\right)(y)-\mathcal{L}_{\sigma-\epsilon}^{*}\left(\phi_{\sigma}\right)(y)\right) N_{\sigma-\epsilon}(y) d y
\end{aligned}
$$

Using (2.7), we have

$$
\left(\mathcal{L}_{\sigma}^{*}-\mathcal{L}_{\sigma-\epsilon}^{*}\right)(g)=B(y)(g(y \sigma)-g(y \sigma-y \epsilon)+g(y(1-\sigma))-g(y(1-\sigma)+y \epsilon)) .
$$


Thus we find

$$
\begin{gathered}
(\lambda(\sigma)-\lambda(\sigma-\epsilon))\left[1-\int_{0}^{\infty}\left(\phi_{\sigma-\epsilon}(y)-\phi_{\sigma}(y)\right) N_{\sigma-\epsilon}(y) d y\right]= \\
\int_{0}^{\infty} B(y)\left(\phi_{\sigma}(y \sigma)-\phi_{\sigma}(y \sigma-y \epsilon)+\phi_{\sigma}(y(1-\sigma))-\phi_{\sigma}(y(1-\sigma)+y \epsilon)\right) N_{\sigma-\epsilon} d y
\end{gathered}
$$

which can be written also as

$$
\begin{gathered}
(\lambda(\sigma)-\lambda(\sigma-\epsilon))\left[1-\int_{0}^{\infty}\left(\phi_{\sigma-\epsilon}(y)-\phi_{\sigma}(y)\right) N_{\sigma-\epsilon}(y) d y\right]= \\
\int_{0}^{\infty} B(y)\left(\int_{y(\sigma-\epsilon)}^{y \sigma} \frac{\partial}{\partial y} \phi_{\sigma}(s) d s+\int_{y(1-\sigma+\epsilon)}^{y(1-\sigma)} \frac{\partial}{\partial y} \phi_{\sigma}(s) d s\right) N_{\sigma-\epsilon} d y .
\end{gathered}
$$

Finally, dividing by $\epsilon$, we have

$$
\frac{\lambda(\sigma)-\lambda(\sigma-\epsilon)}{\epsilon}=\frac{\int_{0}^{\infty} B(y)\left(\int_{y(\sigma-\epsilon)}^{y \sigma} \frac{\partial}{\partial y} \phi_{\sigma}(s) \frac{d s}{\epsilon}+\int_{y(1-\sigma+\epsilon)}^{y(1-\sigma)} \frac{\partial}{\partial y} \phi_{\sigma}(s) \frac{d s}{\epsilon}\right) N_{\sigma-\epsilon} d y}{\left(1-\int_{0}^{\infty}\left(\phi_{\sigma-\epsilon}(y)-\phi_{\sigma}(y)\right) N_{\sigma-\epsilon}(y) d y\right)} .
$$

Thus, using [22], Lemma 2.3 and the Lebesgue dominated convergence theorem, we pass to the limit in (3.12) as $\epsilon \rightarrow 0$. Therefore the function $\lambda(\sigma)$ is differentiable. Moreover, passing to the limit, we obtain (2.9).

\section{Proof of the convexity of the dual eigenfunction}

In this section, we are interested in global conditions on the birth rate $B$ such that the dual function $\phi_{\sigma}($.$) is convex (resp. concave) on the real line. We prove here Theorem 2.7.$

I - Assume that (2.1) holds. Using (1.2), we have,

$$
\phi_{\sigma}(y)=C t e e^{\lambda(\sigma) y}, \quad \forall y \in[0, a],
$$

where $\phi_{\sigma}$ is convex on $[0, \sigma b] \subset[0, a]$ for all $\sigma$ such that $\sigma b<a$.

II - Assume that (2.2) holds. Then we first prove the concavity of the dual eigenfunction in a neighborhood of $y=0$ and we extend the property of concavity to $y \in\left[0, \sigma_{0} b\right]$.

We notice that $\phi_{\sigma}(0)>0, \frac{\partial \phi_{\sigma}}{\partial y}(0)=\left(\lambda_{\sigma}-B(0)\right) \phi_{\sigma}(0)<0$ since $B$ is decreasing (and non constant) and $\lambda_{\sigma}<B(0)=\sup B$. Moreover, assumption (2.2) implies

$$
\frac{\partial^{2} \phi_{\sigma}}{\partial y^{2}}(0)<0
$$


Using

$$
-\frac{\partial \phi_{\sigma}}{\partial y}+\left(B(y)+\lambda_{\sigma}\right) \phi_{\sigma}(y)=B(y)\left(\phi_{\sigma}(\sigma y)+\phi_{\sigma}((1-\sigma) y)\right), \quad y \geq 0
$$

we find,

$$
\begin{gathered}
\frac{\partial^{2} \phi_{\sigma}}{\partial y^{2}}=\lambda_{\sigma} \frac{\partial}{\partial y} \phi_{\sigma}(y)-\frac{B^{\prime}(y)}{B(y)}\left(-\frac{\partial}{\partial y} \phi_{\sigma}+\lambda_{\sigma} \phi_{\sigma}(y)\right) \\
-B(y)\left(\sigma \frac{\partial}{\partial y} \phi_{\sigma}(\sigma y)+(1-\sigma) \frac{\partial}{\partial y} \phi_{\sigma}((1-\sigma) y)-\frac{\partial}{\partial y} \phi_{\sigma}(y)\right), \quad y \geq 0 .
\end{gathered}
$$

Now, assume that there exists $0<\alpha<b \sigma_{0}$ such that

$$
\frac{\partial^{2} \phi_{\sigma}}{\partial y^{2}}(y)<0, \quad y \in\left[0, \alpha\left[, \quad \frac{\partial^{2} \phi_{\sigma}}{\partial y^{2}}(\alpha)=0\right.\right.
$$

and

$$
\frac{\partial \phi_{\sigma}}{\partial y}(y)<0, \quad y \in[0, \alpha[
$$

Then we have

$$
-B(y)\left(\sigma \frac{\partial}{\partial y} \phi_{\sigma}(\sigma y)+(1-\sigma) \frac{\partial}{\partial y} \phi_{\sigma}((1-\sigma) y)-\frac{\partial}{\partial y} \phi_{\sigma}(y)\right)<0, \quad y \in[0, \alpha]
$$

and

$$
\begin{gathered}
\frac{\partial \phi_{\sigma}}{\partial y}(y)<\frac{\partial \phi_{\sigma}}{\partial y}(0)=\left(\lambda_{\sigma}-B(0)\right) \phi_{\sigma}(0)<0, \quad y \in[0, \alpha] \\
0<\phi_{\sigma}(y)<\phi_{\sigma}(0), \quad y \in[0, \alpha] .
\end{gathered}
$$

Assumption (2.2) implies

$$
\lambda_{\sigma}+\frac{B^{\prime}(y)}{B(y)} \geq \lambda_{\sigma}+\frac{\lambda_{\sigma}\left(\lambda_{\sigma}-B(0)\right)}{B(0)}=\frac{\lambda_{\sigma}^{2}}{B(0)} \geq 0, \quad \forall y \in\left[0, b \sigma_{0}\right] .
$$

Thus we obtain

$$
\begin{gathered}
\frac{\partial^{2} \phi_{\sigma}}{\partial y^{2}}(y)<\left[\left(\lambda_{\sigma}+\frac{B^{\prime}(y)}{B(y)}\right)\left(\lambda_{\sigma}-B(0)\right)-\frac{B^{\prime}(y)}{B(y)} \lambda_{\sigma}\right] \phi_{\sigma}(0), \quad y \in[0, \alpha], \\
\frac{\partial^{2} \phi_{\sigma}}{\partial y^{2}}(y)<\left[\lambda_{\sigma} \frac{\lambda_{\sigma}-B(0)}{B(0)}-\frac{B^{\prime}(y)}{B(y)}\right] \phi_{\sigma}(0) B(0), \quad y \in[0, \alpha],
\end{gathered}
$$

and finally, we have

$$
\frac{\partial^{2} \phi_{\sigma}}{\partial y^{2}}(y)<0, \quad y \in[0, \alpha]
$$

Therefore, the strict inequalities (4.1) and (4.2) are satisfied on $\left[0, b \sigma_{0}\right]$ and $\phi_{\sigma}$ is a concave function on $\left[0, \sigma_{0} b\right]$ for all $\sigma \in\left[1 / 2, \sigma_{0}\right]$. 


\section{Extension to a more general cell division model}

In this section we extend the main results to a more general model of cell division. More precisely, in Section 5.1, we generalize the results to the homogeneous cell division (see [22]) and in Section 5.2 , we allow cells to have non constant rate of division.

\subsection{Homogeneous cell division}

A cell of size $y$ may give birth to a cell of size $y s, s \in[0,1]$, with "probability" $\Theta(s)$ where

$$
\begin{aligned}
& \Theta(s)=\Theta(1-s), \\
& \int_{0}^{1} s \Theta(s) d s=1, \quad \int_{0}^{1} \Theta(s) d s=2, \\
& \left.\int_{\eta}^{1} \Theta(s) d s \leq 1, \text { for } \eta \in\right] 0,1[.
\end{aligned}
$$

Equation (5.1) means that the probability $\Theta$ must be symmetric with respect to $s=1 / 2$ (the symmetric division). Equation (5.2) implies the conservation of "size" after division and fix the average number of childs after division to 2 . The density of cell population $n$ satisfies the main equation

$$
\frac{\partial}{\partial t} n(t, y)+\frac{\partial}{\partial y} n(t, y)+B(y) n(t, y)=\int_{0}^{1} \Theta(s) B(y / s) n(t, y / s) \frac{d s}{s},
$$

Again, we prove, using General Relative Entropy method that the asymptotic behavior of such an evolution equation is given by the following eigenproblem

$$
\left\{\begin{array}{l}
\frac{\partial}{\partial y} N(y)+[\lambda+B(y)] N(y)=\int_{0}^{1} \Theta(s) B(y / s) N(y / s) \frac{d s}{s}, \\
-\frac{\partial}{\partial y} \phi(y)+\lambda \phi(y)=B(y)\left[\int_{0}^{1} \Theta(s) \phi(y s) d s-\phi(y)\right], \\
N(y=0)=0, \quad N, \phi \geq 0, \quad \int_{0}^{\infty} N(y) \phi(y) d y=1, \quad \phi(0)=1 .
\end{array}\right.
$$

Definition 5.1. We call $\lambda_{\Theta}$ the first eigenvalue associated to eigenproblem (5.5) and $\lambda_{\text {sym }}$ the eigenvalue associated to $\Theta(s)=\delta_{s=1 / 2}$.

Then we have the following theorem.

Theorem 5.2. Assume that (2.1) (resp. (2.2)) holds and

$$
\text { ConvHull }(\operatorname{Supp} \Theta):=\text { Convex Hull }(\operatorname{Supp} \Theta) \subset[1-a / b, a / b] \quad\left(\operatorname{resp} \cdot\left[1-\sigma_{0}, \sigma_{0}\right]\right) .
$$

Then we have

$$
\lambda_{\Theta} \geq \lambda_{\text {sym }}, \quad\left(\text { resp. } \lambda_{\Theta} \leq \lambda_{\text {sym }}\right) .
$$


Thus, the less (resp. the 'best') fitted division is the symmetric division.

Proof of Theorem 5.2 Under assumption (2.1) (resp. (2.2)), we directly obtain that the dual eigenfunctions are convex (resp. concave) on

$$
\text { ConvHull }(\operatorname{Supp} \Theta) . \text { Supp } B:=\{r y, r \in \operatorname{ConvHull}(\operatorname{Supp} \Theta), y \in \operatorname{Supp} B\} \text {, }
$$

for all $\Theta$ that satisfies (5.1)-(5.3) and (5.6). We use a min-max principle to conclude the proof of the theorem.

\section{Step 1. Min-max method:}

Let

$$
\mathcal{L}(f):=-\frac{\partial f}{\partial y}(.)-B(.) f(t, .)+\int_{0}^{1} \Theta(s) B(. / s) f(. / s) \frac{d s}{s},
$$

and the dual operator,

$$
\mathcal{L}^{*}(f):=\frac{\partial f}{\partial y}(.)-B(.) f(t, .)+B(.)\left[\int_{0}^{1} \Theta(s) f(. s) d s-f(.)\right] .
$$

We have the min-max lemma (see Section 3). Lemma 5.3. Assume that there exists $(N, \lambda, \phi)$ solution to (5.5). Then we have for all $\Theta$,

$$
\lambda_{\Theta}=\max _{f \in \Lambda} \min _{g \in \Lambda^{*}} \frac{\langle g, \mathcal{L} f\rangle}{\langle g, f\rangle},
$$

where $\phi \in \Lambda^{*} \subset D_{\text {om }}\left(\mathcal{L}^{*}\right)$ and $N \in \Lambda \subset D_{\text {om }}(\mathcal{L})$.

Step 2. Application to the homogeneous cell division: Let

$$
\mathcal{L}_{\text {sym }}(f):=-\frac{\partial f}{\partial y}(.)-B(.) f(t, .)+4 B(2 .) f(2 .),
$$

and the dual operator,

$$
\mathcal{L}_{\text {sym }}^{*}(f):=\frac{\partial f}{\partial y}(.)-B(.) f(t, .)+B(.)[2 f(. / 2)-f(.)] .
$$

Lemma 5.4. Assuming (2.1) (resp. 2.2)), we have for all $\Theta$ satisfying (5.1)-(5.3) and (5.6),

$$
\lambda_{\Theta} \geq \lambda_{\text {sym }} \quad\left(\text { resp } \leq \lambda_{\text {sym }}\right) .
$$

Proof. We have shown that for all $\Theta$ satisfying (5.1)-(5.3) and (5.6), and $B$ satisfying (2.1) (resp. $2.2)$ ), the dual functions $\phi$ (and in particular $\phi_{\text {sym }}$ ) are convex (resp. concave) functions on ConvHull $(\operatorname{Supp} \Theta)$.Supp B. Thus, we can choose $\Lambda^{*}$ as the set of positive convex (resp. concave) functions on ConvHull $(\operatorname{Supp} \Theta)$.Supp B. We have

$$
\mathcal{L}^{*}(f)-\mathcal{L}_{\text {sym }}^{*}(f)=B(.) \int_{0}^{1} \Theta(s)[f(s .)-f(. / 2)] d s, \quad \forall f \in \Lambda^{*},
$$


which can be rewritten as

$$
\mathcal{L}^{*}(f)-\mathcal{L}_{\text {sym }}^{*}(f)=2 B(.) \int_{1 / 2}^{1} \Theta(s)\left[\frac{f(s .)+f((1-s) .)}{2}-f(. / 2)\right] d s, \quad \forall f \in \Lambda^{*} .
$$

Since $f \in \Lambda^{*}$ is a convex (resp. concave) function on $\operatorname{ConvHull}(\operatorname{Supp} \Theta)$.Supp B, then we have $\frac{f(s .)+f((1-s) .)}{2}-f(. / 2) \geq 0$ (resp. $\left.\leq 0\right)$, and

$$
\mathcal{L}^{*}(f)-\mathcal{L}_{\text {sym }}^{*}(f) \geq 0, \quad \forall f \in \Lambda^{*},(\text { resp. } \leq 0)
$$

Thus, using the min-max Lemma 5.3, we have $\lambda(\Theta) \geq \lambda_{\text {sym }}$ (resp. $\lambda(\Theta) \leq \lambda_{\text {sym }}$ ).

\subsection{Cell division model with non constant speed rate}

In this section we consider the cell division equation

$$
\frac{\partial}{\partial t} n(t, y)+\frac{\partial}{\partial y} V(y) n(t, y)+B(y) n(t, y)=\int_{0}^{1} \Theta(s) B(y / s) n(t, y / s) d s / s,
$$

where the birth rate satisfies Supp $B(y) \subset[a, b]$ and the speed rate $V(y)=y^{\mu}$. As above, we prove, using General Relative Entropy method that the asymptotic behavior of such an evolution equation is given by the following eigenproblem

$$
\left\{\begin{array}{l}
\frac{\partial}{\partial y}(V(y) N(y))+[\lambda+B(y)] N(y)=\int_{0}^{1} \Theta(s) B(y / s) N(y / s) d s / s, \quad y \geq 0, \\
-V(y) \frac{\partial}{\partial y} \phi(y)+[\lambda+B(y)] \phi(y)=B(y) \int_{0}^{1} \Theta(s) \phi(y s) d s, \quad y \geq 0, \\
N(0)=0, \quad N \geq 0, \quad \phi \geq 0, \quad \int N(y) \phi(y) d y=1 .
\end{array}\right.
$$

If $\lambda_{\text {sym }} \neq 0$ and one of these conditions holds

$$
\begin{gathered}
0<\mu<1 \text { and } 0<\lambda_{\text {sym }}<\mu a^{\mu-1}, \\
\mu>1 \text { and } \lambda_{\text {sym }}>\mu a^{\mu-1}
\end{gathered}
$$

or

$$
\lambda_{\text {sym }}<0,
$$

then we have the following theorem. 
Theorem 5.5. Assume that $\Theta$ satisfies (5.1)-(5.3) and (5.6), and $\mu$ satisfies (5.15) (resp. (5.16) or (5.17)). Then we have

$$
\sup _{\Theta} \lambda_{\Theta} \leq \lambda_{\text {sym }} \quad\left(\text { resp. } \quad \geq \lambda_{\text {sym }}\right)
$$

Here, we only have to prove the convexity (resp. the concavity) of the dual eigenfunction in (5.14). First, we recall results on the existence of a solution to the eigenproblem. Then, we use the differentiation method in order to find the variation of the invasive parameter with respect to $\Theta$ by proving that the solution $\phi_{\Theta}$ to the dual problem is convex (or concave).

Step 1. Existence and uniqueness of the solution to the eigenproblem (5.14). We have the following lemma. Lemma 5.6. Under the assumption $\mu>-1$, there exists a solution $\left(N_{\sigma}, \lambda(\sigma), \phi_{\sigma}\right)$ to (2.5). We refer to [22] for the proof of this result.

Step 2. Corollary of the min-max method Using the proof of Theorem 5.2 and Lemmas 5.3 and 5.4, we have the following lemma.

Lemma 5.7. If $\phi_{\sigma}$ is a convex (resp. concave) function on $[1 / 2-\eta, 1 / 2+\eta]$.Supp $B=\sigma$ Supp B with $\Theta=\delta_{\sigma}+\delta_{1-\sigma}$ for $\sigma \in[1 / 2-\eta, 1 / 2+\eta]$, then

$$
\left.\forall 1 / 2-\eta<\sigma_{0}<\sigma_{1}<1 / 2+\eta, \quad \lambda_{\sigma_{0}}<\lambda_{\sigma_{1}} \text { (resp. }>\lambda_{\sigma_{1}}\right) .
$$

Moreover, if we assume that the probabilities $\Theta$ satisfy (5.1)-(5.3) and (5.6), and the dual eigenfunction $\phi$ is convex (resp. concave) on ConvHull (Supp $\Theta)$. Supp B, then we have

$$
\inf _{\Theta} \lambda_{\Theta}=\lambda_{\text {sym }} \quad\left(\text { resp. } \sup _{\Theta} \lambda_{\Theta}=\lambda_{\text {sym }}\right) \text {. }
$$

Proof. Indeed, we have,

$$
\mathcal{L}_{\sigma_{0}}^{*}(f)-\mathcal{L}_{\sigma_{1}}^{*}(f)=B(.)\left[f\left(\sigma_{0} .\right)+f\left(\left(1-\sigma_{0}\right) .\right)-f\left(\sigma_{1} .\right)-f\left(\left(1-\sigma_{1}\right) .\right)\right] d s, \quad \forall f \in \Lambda^{*},
$$

where $\Lambda^{*}$ is the set of positive convex (resp. concave) functions on ConvHull(Supp $\left.\Theta\right)$.Supp B. Since $1 / 2-\eta<\sigma_{0}<\sigma_{1}<1 / 2+\eta$, then we find that the constant $t$ given by the equality

$$
\left.t=\frac{\sigma_{0}+\sigma_{1}-1}{2 \sigma_{1}-1} \in\right] 0,1[
$$

satisfies $\sigma_{0}=t \sigma_{1}+(1-t) \sigma_{1}$ and $1-\sigma_{0}=t \sigma_{1}+(1-t) \sigma_{1}$. We have

$$
\begin{gathered}
f\left(\sigma_{0} y\right)+f\left(\left(1-\sigma_{0}\right) y\right)-f\left(\sigma_{1} y\right)-f\left(\left(1-\sigma_{1}\right) y\right)= \\
{\left[f\left(\sigma_{0} y\right)-t f\left(\sigma_{1} y\right)-(1-t) f\left(\left(1-\sigma_{1}\right) y\right)\right]+\left[f\left(\left(1-\sigma_{0}\right) y\right)-t f\left(\left(1-\sigma_{1}\right) y\right)-(1-t) f\left(\sigma_{1} y\right)\right]}
\end{gathered}
$$

with $f$ positive convex (resp. concave) function and $t \in] 0,1[$. Thus, we find that

$$
\mathcal{L}_{\sigma_{0}}^{*}(f)-\mathcal{L}_{\sigma_{1}}^{*}(f) \leq 0 \quad(\text { resp. } \geq 0), \quad \forall f \in \Lambda^{*} .
$$


We conclude using the extension of the min-max Lemma 5.3.

Next, we assume that for all $\Theta$ satisfying (5.1)-(5.3) and (5.6), the dual functions $\phi$ (and in particular $\phi_{\text {sym }}$ ) are convex (resp. concave) functions on ConvHull $(\operatorname{Supp} \Theta)$.Supp $B=[1-$ $\sigma, \sigma] . S u p p$ B. Thus, we can choose, in Lemma 5.3, $\Lambda^{*}$ as the set of positive convex (resp. concave) functions on $[1-\sigma, \sigma]$.Supp $B$. We have

$$
\mathcal{L}^{*}(f)-\mathcal{L}_{\text {sym }}^{*}(f)=B(.) \int_{0}^{1} \Theta(s)[f(s .)-f(. / 2)] d s, \quad \forall f \in \Lambda^{*},
$$

which can be rewritten as

$$
\mathcal{L}^{*}(f)-\mathcal{L}_{\text {sym }}^{*}(f)=2 B(.) \int_{1 / 2}^{1} \Theta(s)\left[\frac{f(s .)+f((1-s) .)}{2}-f(. / 2)\right] d s, \quad \forall f \in \Lambda^{*} .
$$

Since $f \in \Lambda$ is a positive convex (resp. concave) function, then we have $\frac{f(s .)+f((1-s) .)}{2}-f(. / 2) \geq 0$ (resp. $\leq 0)$

$$
\mathcal{L}^{*}(f)-\mathcal{L}_{\text {sym }}^{*}(f) \geq 0(\text { resp. } \leq 0), \quad \forall f \in \Lambda^{*} .
$$

Thus, using the min-max Lemma 5.3, we have $\lambda(\Theta) \geq \lambda_{\text {sym }}$ (resp. $\lambda(\Theta) \leq \lambda_{\text {sym }}$ ).

Step 3. Concavity of the dual eigenfunction We have

$$
\phi^{\prime \prime}(y)=\frac{\lambda^{2}}{V(y)^{2}}\left(1-V^{\prime} / \lambda\right), \quad y \in \operatorname{Supp} B
$$

with $V(y)=y^{\mu}$ and Supp $B=[a, b]$. Therefore, we have clearly that assumptions (5.16) or (5.17) lead to $\phi^{\prime \prime}(y)>0$ on ConvHull $(\operatorname{Supp} \Theta)$.Supp B and condition (5.15) leads to $\phi^{\prime \prime}(y)<0$ on ConvHull(Supp $\Theta)$.Supp B.

Step 4. Conclusion Thus, using Lemma 5.7 and step 3, we prove the theorem.

\section{Simple application of the differentiation and the min-max methods}

In this section, in order to apply both methods, we focus our attention on a simple model of McKendrick-VonFoerster in which cells are structured by their age (see for instance [8, 12, 16, 23, $31])$. The density of the population of age $a$ at time $t$ is denoted by $n(t, a)$ and follows the renewal equation

$$
\left\{\begin{array}{l}
\frac{\partial}{\partial t} n(t, a)+\frac{\partial}{\partial a} n(t, a)+d(a) n(t, a)=0, \\
n(t, 0)=\int_{0}^{\infty} B(a) n(t, a) d a
\end{array}\right.
$$


where $B$ is the growth rate and $d$ the death rate. As above, the GRE gives the asymptotic behavior of $n(t,.) \sim \operatorname{cst} N(.) e^{\lambda t}$, where $(N, \lambda, \phi)$ is a solution to the eigenproblem

$$
\left\{\begin{array}{l}
\frac{\partial}{\partial a} N(a)+d(a) N(a)+\lambda N(a)=0, \quad N(0)=\int_{0}^{\infty} B(a) N(a) d a \\
-\frac{\partial}{\partial a} \phi(a)+d(a) \phi(a)+\lambda \phi(a)=B(a) \phi(0), \\
\int_{0}^{\infty} N(a) \phi(a) d a=1 .
\end{array}\right.
$$

The relevance of this model is its simplicity, contrary to the cell division model (1.1). We can easily compute $(N, \lambda, \phi)$. Indeed, if there exists $\lambda$ such that

$$
\int_{0}^{\infty} B\left(a^{\prime}\right) e^{-\int_{0}^{a^{\prime}}(d(s)+\lambda) d s} d a^{\prime}=1
$$

then we have

$$
\left\{\begin{array}{l}
N(a)=e^{-\int_{0}^{a}(d(s)+\lambda) d s} \\
\phi(a)=\int_{a}^{\infty} B\left(a^{\prime}\right) e^{-\int_{a}^{a^{\prime}}(d(s)+\lambda) d s} d a^{\prime}
\end{array}\right.
$$

Let

$$
\begin{gathered}
\mathcal{L}(f)=-\left[\frac{\partial}{\partial a} f(a)+d(a) f(a)\right]+\delta_{a=0} \int_{0}^{\infty} B\left(a^{\prime}\right) f\left(a^{\prime}\right) d a^{\prime}, \\
\mathcal{L}^{*}(g)=-\left[-\frac{\partial}{\partial a} g(a)+d(a) g(a)\right]+g(0) B(a) .
\end{gathered}
$$

Thus we have the following lemma.

Lemma 6.1. Assume that $B, d \in L^{\infty}([0, \infty[,[0, \infty[)$ and Supp $B \subset[0, A]$ is compact. Then we have the existence of a solution $(N, \lambda, \phi)$ to eigenproblem (6.2). Moreover, we have,

$$
\begin{gathered}
\mathcal{L}(f): L^{2}([0, A]) \mapsto \mathcal{M}([0, A]), \\
\mathcal{L}^{*}(f): C_{0}^{0}\left(\left[0, \infty[) \mapsto L^{2}([0, \infty[),\right.\right.
\end{gathered}
$$

where $\mathcal{M}([0, A])$ is the set of a bounded measure on $[0, A]$ and

$$
N \in C_{b}^{0}([0, A]), \quad N \leq 1, \quad \operatorname{Supp} \phi \subset[0, A], \quad \phi \leq \int_{0}^{A} B(s) d s e^{\lambda A} .
$$

Therefore (3.1)-(3.3) are satisfied for $\mathcal{B}=L^{2}$ and $\mathcal{C}=C_{0}^{0}([0, \infty[)$. 


\section{Evolution of the invasive parameter $\lambda$ with respect to the growth of the death rate $d$ or the birth rate $B$.}

The aim of this part is to study the evolution of the first eigenvalue $\lambda$ when the death rate (resp. the birth rate) increases.

Growth of death rate. Let $n_{1}$ (resp. $n_{2}$ ) be the density of the population satisfying (6.1) with the death rate $d=d_{1}$ (resp. $d=d_{2}$ ) that verifies the assumptions of Lemma 6.1.

Let $\lambda_{1}$ (resp. $\lambda_{2}$ ) be the invasive parameter, that is the eigenvalue, associated to the first population $n_{1}$ (resp. the second population $n_{2}$ )

Lemma 6.2. Assume that $0 \leq d_{1} \leq d_{2}$. Then $\lambda_{2} \leq \lambda_{1}$. Thus, the population $n_{2}$ is less fitted than the population $n_{1}$.

Proof. Here we can use both methods. Indeed, let

$$
\begin{aligned}
& \mathcal{L}_{1}(f)=-\left[\frac{\partial}{\partial a} f(a)+d_{1}(a) f(a)\right]+\delta_{a=0} \int_{0}^{\infty} B\left(a^{\prime}\right) f\left(a^{\prime}\right) d a^{\prime}, \\
& \mathcal{L}_{2}(f)=-\left[\frac{\partial}{\partial a} f(a)+d_{2}(a) f(a)\right]+\delta_{a=0} \int_{0}^{\infty} B\left(a^{\prime}\right) f\left(a^{\prime}\right) d a^{\prime},
\end{aligned}
$$

then $\mathcal{L}_{1}(f)=\mathcal{L}_{2}(f)+\left(d_{2}-d_{1}\right)(a) f(a) \geq \mathcal{L}_{2}(f)$ for all $f \geq 0$ and thus, using Lemma 3.2, we obtain that $\lambda_{1} \geq \lambda_{2}$.

Remark. The differentiation method could be used to prove the lemma. Indeed, let

$$
\mathcal{L}_{p}^{*}(f)=-\left[-\frac{\partial}{\partial a} f(a)+d_{p}(a) f(a)\right]+B(a) f(0),
$$

with $d_{p}=p d_{2}+(1-p) d_{1}$ and $p \in[0,1]$. Then

$$
\mathcal{L}_{p}^{*}(f)-\mathcal{L}_{q}^{*}(f)=\left[d_{q}(a)-d_{p}(a)\right] f(a)=(p-q)\left(d_{1}-d_{2}\right),
$$

and so (3.8) is satisfied. Moreover, the implicit form of $\lambda(p)$ and the explicit form of $N_{p}$ imply directly condition (3.7). Therefore, we have,

$$
\frac{d}{d p} \lambda(p)=\int_{0}^{\infty}\left(d_{1}-d_{2}\right)(a) \phi_{p}(a) N_{p}(a) d a \leq 0,
$$

and we find $\lambda_{2}=\lambda(1) \leq \lambda(0)=\lambda_{1}$.

Growth of birth rate. Let $n_{1}$ (resp. $n_{2}$ ) be the density of the population satisfying (6.1) with the birth rate $B=B_{1}$ (resp. $B=B_{2}$ ) that verifies the assumptions of Lemma 6.1.

Let $\lambda_{1}$ (resp. $\lambda_{2}$ ) be the invasive parameter, i.e., eigenvalue, associated to the first population $n_{1}$ (resp. the second population $n_{2}$ )

Lemma 6.3. Assume that $0 \leq B_{1} \leq B_{2}$. Then $\lambda_{1} \leq \lambda_{2}$. Thus, the population $n_{2}$ is better fitted 
than the population $n_{1}$.

Proof. Here we can use both methods. Indeed, let

$$
\begin{aligned}
& \mathcal{L}_{1}(f)=-\left[\frac{\partial}{\partial a} f(a)+d(a) f(a)\right]+\delta_{a=0} \int_{0}^{\infty} B_{1}\left(a^{\prime}\right) f\left(a^{\prime}\right) d a^{\prime}, \\
& \mathcal{L}_{2}(f)=-\left[\frac{\partial}{\partial a} f(a)+d(a) f(a)\right]+\delta_{a=0} \int_{0}^{\infty} B_{2}\left(a^{\prime}\right) f\left(a^{\prime}\right) d a^{\prime} .
\end{aligned}
$$

Then $\mathcal{L}_{1}(f)=\mathcal{L}_{2}(f)+\delta_{a=0} \int_{0}^{\infty}\left(B_{1}\left(a^{\prime}\right)-B_{2}\left(a^{\prime}\right)\right) f\left(a^{\prime}\right) d a^{\prime} \leq \mathcal{L}_{2}(f)$ for all $f \geq 0$ and thus, using Lemma 3.2, we obtain that $\lambda_{1} \leq \lambda_{2}$.

Remark. The differentiation method could be used to prove the lemma. Indeed, let

$$
\mathcal{L}_{p}^{*}(f)=-\left[-\frac{\partial}{\partial a} f(a)+d(a) f(a)\right]+B_{p}(a) f(0),
$$

with $B_{p}=p B_{2}+(1-p) B_{1}$ and $p \in[0,1]$. Then (as above)

$$
\frac{d}{d p} \lambda(p)=\int_{0}^{\infty}\left(B_{2}-B_{1}\right)(a) \phi_{p}(0) N_{p}(a) d a \geq 0,
$$

and we find $\lambda_{2}=\lambda(1) \geq \lambda(0)=\lambda_{1}$.

We notice that both examples give the same results using the min-max method or the differentiation method. Here, we give an example which is more difficult to study with the min-max method.

\section{Evolution of the invasive parameter $\lambda$ with respect to a complex variation of the death rate $d$.}

Child Vs Oldness death. Let $n_{p}$ be the density of the population satisfying (6.1) with the death rate $d_{p}(y)=1_{y \in[p, p+1]} d(y)$ that verifies the assumptions of Lemma 6.1. More precisely, the death rate $d_{p}$ has its support on the interval $[p, p+1]$, which means that for small $p$, only childs die and for large $p$, only old individuals die. Let $\lambda(p)$ be the invasive parameter, that is the eigenvalue, associated to the population $n_{p}$. In this part, we assume that

$$
\text { Supp } B=[\alpha, \beta] \subset[0, \infty[,
$$

and

$$
d \in C^{1}([0, \infty[) \text { decreases on }[0, \beta-1] .
$$

Then we have the following result.

Lemma 6.4. Assume that (6.5) and (6.6) are satisfied. Then $\lambda(p)$ is increasing, that is the juvenile death rate is less fitted than 'oldness' death rate.

Proof. Here we can use only the differentiation method. Indeed, let

$$
\mathcal{L}_{p}^{*}(f)=-\left[-\frac{\partial}{\partial a} f(a)+d_{p}(a) f(a)\right]+B(a) f(0) .
$$


Then for $d_{p}(y)=1_{y \in[p, p+1]} d(y)=1_{p \in[y-1, y]} d(y)$ we have

$$
-\frac{\partial}{\partial p} d_{p}(y)=-\delta_{p=y-1} d(p+1)+\delta_{p=y} d(p)
$$

and so, we find

$$
\frac{d}{d p} \lambda(p)=d(p) \phi_{p}(p) N_{p}(p)-d(p+1) \phi_{p}(p+1) N_{p}(p+1)
$$

Therefore, using (6.4),we obtain,

$$
\frac{d}{d p} \lambda(p)=-d(p+1) \int_{p+1}^{\infty} B\left(a^{\prime}\right) e^{-\int_{0}^{a^{\prime}}(d(s)+\lambda) d s} d a^{\prime}+d(p) \int_{p}^{\infty} B\left(a^{\prime}\right) e^{-\int_{0}^{a^{\prime}}(d(s)+\lambda) d s} d a^{\prime},
$$

and so, we have,

$$
\frac{d}{d p} \lambda(p)=[d(p)-d(p+1)] \int_{p+1}^{\infty} B\left(a^{\prime}\right) e^{-\int_{0}^{a^{\prime}}(d(s)+\lambda) d s} d a^{\prime}+d(p) \int_{p}^{p+1} B\left(a^{\prime}\right) e^{-\int_{0}^{a^{\prime}}(d(s)+\lambda) d s} d a^{\prime} .
$$

Since $d$ increases (see (6.6)), then $[d(p)-d(p+1)] \int_{p+1}^{\infty} B\left(a^{\prime}\right) e^{-\int_{0}^{a^{\prime}}(d(s)+\lambda) d s} d a^{\prime} \geq 0$ and

$$
\frac{d}{d p} \lambda(p) \geq 0
$$

Therefore, we obtain that $\lambda(p)$ is increasing.

We notice that the min-max method is not well adapted when the growth of the operators $\mathcal{L}_{p}$ with respect to $p$ is hard to prove. As for instance in the McKendrick-Von Foerster model when the death rates cannot be compared. The question of knowing the variation of the invasive parameter with respect to the asymmetry parameter leads to the same remark and therefore, we first use the differentiation method.

\section{Discussion}

In this paper we have studied the variation of the Malthusian growth rate of a cell population due to the variability of the symmetry of the cell division during mitosis [15]. We show that under the assumption of a constant speed rate, equal mitosis is not necessarily the division giving the better growth rate. More precisely, when the cells divide lately, that is for large size relatively to the average size of a dividing cell, then equal mitosis is not the best fitted division. When cells can divide early, then equal mitosis is the best fitted division.

Obviously, this model is a simple one and the conclusion depends on the assumptions such as : - there is no interaction between cells, no competition between species with different asymmetry parameters, 
- the speed rate is constant (or a power of the size) which cannot be the case in general,

- there is no apoptosis in the model.

Nevertheless, the tools we have used to study this problem, that is the differentiation method and the min-max method, seem to be well fitted to study the variation of the Malthusian growth rate with respect to a parameter for various problems (see Section 6).

An interesting question to study is the competition between two species of cells (with two different cell divisions) as for instance for normal and tumoral (due to mutation) cells.

\section{References}

[1] M. Ackermann, S.C. Stearns, U. Jenal. Senescence in a bacterium with asymmetric division. Science, 300 (2003), 1920.

[2] M.G. Barker, R.M. Walmsley. Replicative ageing in the fission yeast Schizosaccharomyces pombe. Yeast, 15 (1999), 1511-1518.

[3] H. Berestycki, F. Hamel. Front propagation in periodic excitable media. Comm. Pure Appl. Math., 55 (2002), 949-1032.

[4] H. Berestycki, F. Hamel, N. Nadirashvili. Elliptic eigenvalue problems with large drift and applications to non linear propagation phenomena. Comm. Part. Diff. Equ., 53 (2005), 451480.

[5] J. Bertoin, A.V. Gnedin. Asymptotic laws for nonconservative self-similar fragmentations. Electron. J. Probab., 9 (2004), No. 19, 575-593.

[6] M. Breitenbach, F. Madeo, P. Laun, G. Heeren, S. Jarolim, K-U. Frohlich, S. Wissing, A. Pichova. Yeast as a model for ageing and apoptosis research. Topics in Current Genetics, Springer Berlin Heidelberg, 3 (2003), 61-97.

[7] T.H. Brummendorf, W. Dragowska, J.M. Zijlmans, G. Thornbury, P.M. Lansdorp. Asymmetric cell divisions sustain long-term hematopoiesis from single-sorted human fetal liver cells. J. Exp. Med., 188 (1998), No. 6, 1117-1124.

[8] J.M. Cushing. An Introduction to Structured Population Dynamics. SIAM Philadelphia, 1998.

[9] E.J. Dens, K. Bernaerts, A.R. Standaert, J.F. Van Impe. Cell division theory and individualbased modeling of microbial lag: part I. The theory of cell division. Int. J. Food. Microbiol., 101 (2005), No 3, 303-318.

[10] O. Diekmann, M. Gyllenberg, H. Huang, M. Kirkilionis, J.A.J. Metz, H.R. Thieme. On the formulation and analysis of general deterministic structured population models. II. Nonlinear theory. J. Math. Biol., 43 (2001), No. 2, 157-189. 
[11] M. Escobedo, S. Mischler, M. Rodriguez Ricard. On self-similarity and stationary problem for fragmentation and coagulation models. Annales de l'Institut Henri Poincare (C) Non Linear Analysis, 22 (2005), No. 1, 99-125.

[12] W. Feller. An Introduction to Probability Theory and Its Applications. Volume I,II. Wiley, New York, 3rd edition, 1968.

[13] N. Fournier, S. Mischler. Exponential trend to equilibrium for discrete coagulation equations with strong fragmentation and without a balance condition. Proc. R. Soc. Lond. Ser. A Math. Phys. Eng. Sci. , 460 (2004), No. 2049, 2477-2486.

[14] R.A. Horn, C.R. Johnson. Matrix Analysis. Cambridge: Cambridge University Press, 1985, Chap 6.3 p372.

[15] S. Huang, P. Law, K. Francis, B.O. Palsson, A.D. Ho. Symmetry of Initial Cell Divisions Among Primitive Hematopoietic Progenitors Is Independent of Ontogenic Age and Regulatory Molecules. Blood, 1999, 94 (1999), No. 8, 2595-2604.

[16] M. Iannelli. Mathematical Theory of Age-Structured Population Dynamics, Giardini Editori, Pisa, 1994.

[17] S.M. Jazwinski, Growing old: Metabolic control and yeast aging. Annu. Rev. Microbiol., 56 (2002), 769-792.

[18] T.B.L. Kirkwood, R. Holliday. The evolution of ageing and longevity. Proc. R. Soc. Lond. B. Biol. Sci., 205 (1979), 531-546.

[19] P. Laurençot. Steady states for a fragmentation equation with size diffusion., Banach Center Publ., 66 (2004), 211-219.

[20] J.A.J. Metz, O. Diekmann. The dynamics of physiologically structured populations., LN in biomathematics 68, Springer-Verlag, 1986.

[21] P. Michel, S. Mischler, B. Perthame. General relative entropy inequality: an illustration on growth models. J. Math. Pures et Appl., 84 (2005), No. 9, 235-1260.

[22] P. Michel. Existence of a Solution to the Cell Division Eigenproblem. Math. Mod. Meth. Appl. Sci., 16 (2006), No. 7, 1125-1153.

[23] S. Mischler, B. Perthame, L. Ryzhik. Stability in a Nonlinear Population Maturation Model. Math. Models Meth. Appl. Sci., 12 (2002), No. 12, 1751-1772.

[24] T. Nystrom. Aging in bacteria. Curr. Opin. Microbiol., 5 (2002), 596-601.

[25] L. Partridge, N.H. Barton. Optimality, mutation and the evolution of ageing. Nature, 362 (1993), 305-311. 
[26] B. Perthame, L. Ryzhik. Exponential decay for the fragmentation or cell-division equation. Journal of Differential Equations, 210 (2005), No. 1, 155-177.

[27] Z. Qu, J.N. Weiss, W.R. MacLellan. Coordination of cell growth and cell division: a mathematical modeling study. Journal of Cell Science, 117 (2004), 4199-4207.

[28] D.A. Sinclair. Paradigms and pitfalls of yeast longevity research. Mech. Ageing Dev., 123 (2002), 857-867.

[29] E.J. Stewart, R. Madden, G. Paul, F. Taddei. Aging and death in an organism that reproduces by morphologically symmetric division. PLoS Biol., 3 (2005), No. 2, 295-300.

[30] M. Sugimoto, T. Yasuda. A Cell Kinetic and Cytological Study on the Asymmetric Cell Division of Thymic Lymphoblasts of the Embryonic Rat. Development, Growth and Differentiation, 23 (1981), No. 6, 639-646.

[31] G.F. Webb. Theory of non-linear age-dependent population dynamics. Monographs and Textbook in Pure and Applied Mathematics, 89, New-York Basel: Marcel Dekker Inc., 1985. 